\title{
The Impact of NA Stalk Deletion on HA Receptor Binding Specificity of Avian H7N9 in China in 2013-14 and Avian H7N7 in Netherlands in 2003
}

\author{
Wei Hu \\ Department of Computer Science, Houghton College, Houghton, NY, USA \\ Email: wei.hu@houghton.edu
}

Received 10 February 2015; accepted 24 February 2015; published 28 February 2015

Copyright (C) 2015 by author and Scientific Research Publishing Inc. This work is licensed under the Creative Commons Attribution International License (CC BY). http://creativecommons.org/licenses/by/4.0/

\section{(c) (i) Open Access}

\begin{abstract}
A novel avian origin influenza H7N9 virus emerged in March 2013 in China and caused severe disease in humans. The high human death rate has caused public health concern and attracted global attention. The influenza viruses have two surface proteins: hemagglutinin (HA) and neuraminidase (NA), with HA facilitating viral entry into a host cell and NA helping new viral particles leave a host cell to infect more host cells. In a previous study published in May 2003, we applied computational analysis to the HA sequences of this new virus. Our findings suggested that the HA of this virus had avian type receptors as its primary binding and human types as secondary binding, which was verified by several subsequent wet lab experiments in later months of 2013. We also showed that the human H7N9 and avian H7N9 in China in 2013 shared the same HA receptor binding patterns. The NA protein of this new virus has a stalk deletion that is associated with virulence and host adaptation. In the present study, we wanted to understand the biological effects of this NA deletion on the HA receptor binding preference of this virus. However, the NA proteins of human H7N9 in China in 2013 all carry a stalk deletion so we lack the NA proteins with no stalk deletion as a control. In this study, we chose instead the NA proteins of avian H7N9 in China in 2013-14 and those of avian H7N7 in Netherlands in 2003 as some of them had a NA stalk deletion and some did not. We sought to employ a computational approach to revealing the impact of this NA stalk deletion on HA receptor binding preference of this virus. Our analysis implied that this deletion in the stalk region of NA enhanced the human receptor binding of avian H7N9 in China in 2013-14 and avian H7N7 in the Netherlands in 2003.
\end{abstract}

\section{Keywords}

H7N9, H7N7, Influenza, Receptor Binding Specificity, Stalk Deletion 


\section{Introduction}

One common pathway of influenza transmission is from wild aquatic birds to poultry, and then to humans. The two viral surface proteins hemagglutinin (HA) and neuraminidase (NA) are critical in the interspecies transmission and adaptation of influenza to a new host. They play complementary roles in virus infection. The HA protein is involved in the viral binding of the sialic acid receptors present on the host cell surface, and the NA protein helps the newly born viruses leave the host cell to infect more other host cells, i.e., it cleaves terminally linked sialic acid from various glycoconjugates on the host cell surface to facilitate the release of progeny virions. The HAs of human influenza tent to bind oligosaccharides that terminate with sialic acid linked to galactose by $\alpha 2$,6-linkages (human type receptors), while the HAs of avian influenza favor those by $\alpha 2,3$-linkages (avian type receptors).

Some avian viruses such as H5N1 and H7N7 were found to infect humans, no human infections with H7N9 viruses were reported until reports from China in March 2013. When avian influenza viruses cross the species boundary to infect humans, they undergo genetic modifications that lead to broader host range. One such common genetic change is deletions in the stalk region of the NA protein and additions of glycosylation sites on the HA protein [1]. Therefore, to understand the deletion in the NA stalk region (positions 30 to 90 for amino acids) and its impact on HA features are important.

Originated from multiple reassortment events, a novel avian influenza A virus of H7N9 subtype emerged in March of 2013 in China and had infected 163 people as of 10 January 2014, of whom 50 died of the severe respiratory infection caused by these viruses [2]. The HA gene this new virus is from an avian virus of duck origin and the NA gene from migratory birds [3]. On 28 February 2003, a highly pathogenic avian influenza A virus subtype H7N7 was isolated in the Netherlands. In the following week, this virus infected 89 humans, of whom 78 had conjunctivitis. A Dutch veterinarian infected by this virus died a week later from respiratory distress [4].

The 2013 human H7N9 viruses in China all carry a deletion in the stalk region of the NA protein whereas the 2003 human H7N7 viruses in the Netherlands do not. The stalk is a structure in the NA protein that separates the enzymatically and antigenically head domain from the hydrophobic domain embedded in the viral membrane. Such deletions in multiple subtypes of NA are known as an indicator of aquatic bird viruses that become adapted to terrestrial poultry and are associated with high virulence [1] [5] [6]. It was demonstrated that NA stalk length affects the biological properties of influenza. NA stalk deletion enhances replication and virulence of influenza virus in eggs, chickens and mice, compared to their equivalents with a long stalk [7]. Therefore, it is desirable to study the advantages conferred by stalk deletion in NA.

However, the NA proteins of human H7N9 in China in 2013-14 all contain this deletion so they cannot be used for this study for lack of a control to serve as a baseline. In this study, we selected instead the NA sequences from avian H7N9 in China in 2013-14 and avian H7N7 in Netherlands in 2003, as some of them contained this deletion but some did not. A computational method was employed to analyze the impact of NA stalk deletion on the HA receptor binding specificity of these viruses.

\section{Materials and Methods}

\subsection{Sequence Data}

The HA and NA protein sequences of avian H7N9 in China from 2013 to 2014 and avian H7N7 in the Netherlands in 2003 were retrieved from the EpiFlu Database (http://platform.gisaid.org of GISAID). We only selected the paired HA and NA sequences that came from the same isolate so the relationship between the two could be used in this study.

\subsection{Informational Spectrum Method}

The informational spectrum method (ISM) is a bioinformatics approach to analyzing protein sequences [8]-[10]. The first step of this technique is to transform the protein sequences into numerical sequences based on electron-ion interaction potential (EIIP) of each amino acid. Then the Discrete Fourier Transform (DFT) is applied to these numerical sequences, and the resulting DFT coefficients are used to produce the energy density spectrum. The informational spectrum (IS) comprises the frequencies and the amplitudes of this energy density spectrum. According to the ISM theory, the peak frequencies of IS of a protein sequence reflect its biological or biochemical functions. The ISM was successfully applied to analyze the receptor binding preference of the HA 
protein in [11]-[13].

In [11] [12] ISM was applied to study the novel H7N9 of avian origin in China in 2013, which discovered that the primary IS frequency is $F(0.285)$ for avian receptor binding and the secondary is $F(0.326)$ for human receptor binding. We also demonstrated that human H7N9 and avian H7N9 in China in 2013 share the same HA receptor binding patterns. Furthermore, mutations that could alter the HA binding preference of this new virus were also found and analyzed. Our findings published in May 2013 were later confirmed by several wet lab experiments in [14]-[20].

\section{Results}

The NA sequences of avian H7N9 in China from 2013 to 2014 and avian H7N7 in 2003 in the Netherlands were divided into two groups respectively: one has the stalk deletion and the other has not. Then we found the matching HA sequence from the same isolate as the NA sequence. In total, there were 54 NA sequences with stalk deletion from avian H7N9 and 26 with no deletion. Also we had 15 NA sequences with stalk deletion from avian H7N7 and 218 with no deletion. As a result of the NA sequence grouping, the HA sequences were grouped accordingly.

The ISM analysis on these HA sequences of avian H7N9 and avian H7N7 is shown in Figure 1. It appeared that the NA stalk deletion had different effects on these two viruses. It reduced the avian binding of avian H7N9 while kept the human binding level intact. But this deletion enhanced human binding of avian H7N7 while maintained its avian binding level. So the consequence of this NA stalk deletion reduced the gap of IS between the primary and secondary frequencies for both avian H7N9 and avian H7N7: from 1.3 to 0.6 in the first case and from 0.14 to 0.05 in the second (Table 1). Thus the NA stalk deletion increased the human binding in both viruses as a net result.

The NA proteins of avian H7N9 carrying the stalk deletion are mostly from chicken with only a few from duck, pigeon, and sparrow, and those in avian H7N7 are all from chicken [21] [22], which implied that chicken viruses are under more pressure to adapt to human hosts than the viruses from other birds. The H7N9 chicken and human isolates all carry a NA stalk deletion, but in the H7N7 case, only part of the chicken isolates have
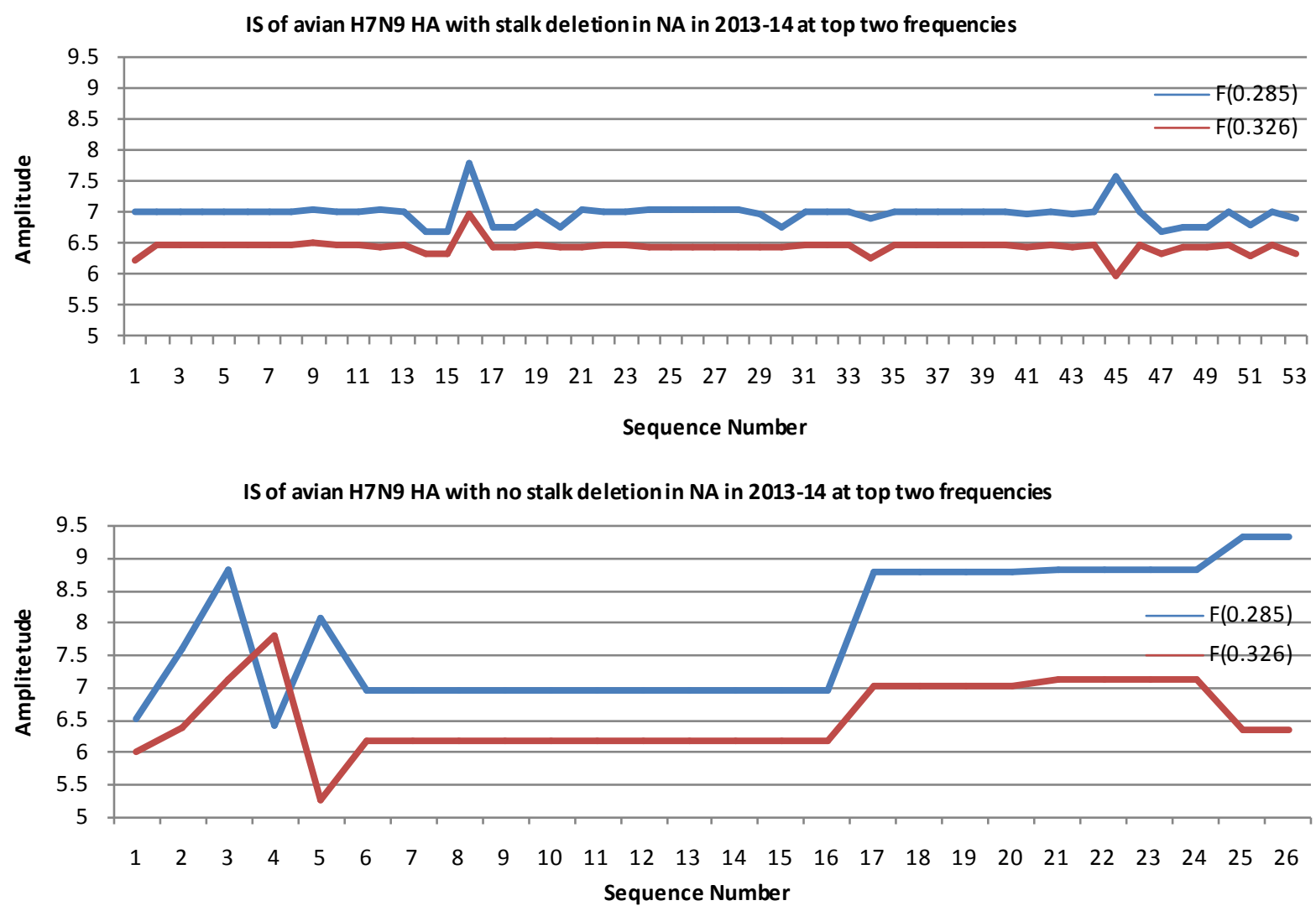

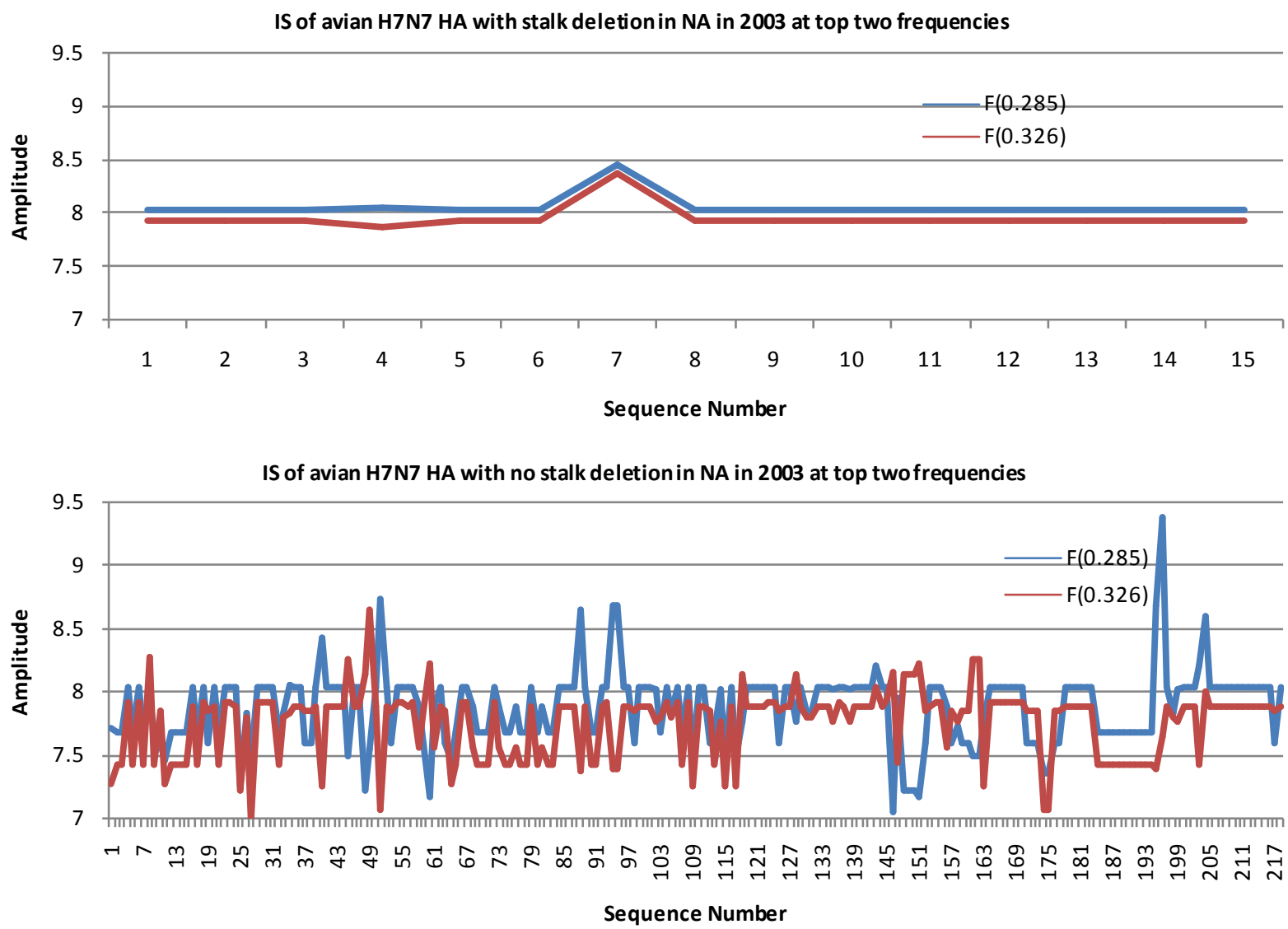

Figure 1. IS of HA at top two frequencies from avian H7N9 in China in 2013-14 and avian H7N7 from Netherlands in 2003 (The scales in the H7N9 or H7N7 plots were the same in order to show the comparison of IS amplitudes between the HAs with NA stalk deletion and those without).

Table 1. Impact of NA stalk deletion on the IS of HA at primary and secondary frequencies.

\begin{tabular}{ccccc}
\hline & \multicolumn{2}{c}{ Avian H7N9 HA } & \multicolumn{2}{c}{ Avian H7N7 HA } \\
\hline & With NA stalk deletion & No NA stalk deletion & With NA stalk deletion & No NA stalk deletion \\
\hline Average IS at F(0.285) & 7 & 7.8 & 8 & 7.9 \\
Average IS at F(0.326) & 6.4 & 6.5 & 7.95 & 7.76 \\
Difference of average IS & 0.6 & 1.3 & 0.05 & 0.14 \\
\hline
\end{tabular}

this deletion. So in this sense, the comparison of HA binding preferences is more direct in avian H7N7 (chicken to chicken) than in avian H7N9 (chicken to other birds). Just looking at the NA stalk deletion, the 2013 H7N9 human viruses in China are more adapted to human hosts than the 2003 human H7N7 in the Netherlands. At the same time, this deletion may also have caused 2013 H7N9 having a higher virulence than 2003 H7N7 as shown in the clinical symptoms [2] [4].

\section{Conclusions}

It is well known that the two surface proteins HA and NA of influenza viruses are key determinants in viral interspecies transmission and host adaptation, during which changes in HA and NA are necessary. One such notable change is the deletion in the stalk region of NA, serving as a genotypic marker of host adaptation. The novel avian origin H7N9 virus discovered in March in China in 2013 has its NA protein containing this deletion. We desired to elucidate the possible effects on the HA receptor binding preference caused by this deletion. However, 
the NA sequences of human H7N9 in China in 2013-14 all have this deletion making them unsuitable for this study as we lack a control.

In this work, we chose instead the NA proteins of avian human H7N9 in China in 2013-14 and avian H7N7 in Netherlands in 2003 as some of them had this NA deletion while some did not. We applied a computational method to study the impact on the HA receptor binding preference caused by the NA stalk deletion. Our findings suggested that the stalk deletion in NA reduced the avian receptor binding in avian H7N9 in China in 2013-14 while keeping its human binding level the same. And the deletion increased the human receptor binding in avian H7N7 in Netherlands in 2003 while maintaining its avian binding level. Taken together, this NA stalk deletion enhanced the human binding of these two avian viruses.

\section{Acknowledgements}

We thank Houghton College for its financial support.

\section{References}

[1] Li, J., Dohna, H.Z., Cardona, C.J., Miller, J. and Carpenter, T.E. (2011) Emergence and Genetic Variation of Neuraminidase Stalk Deletions in Avian Influenza Viruses. PLOS ONE, 6, e14722. http://dx.doi.org/10.1371/journal.pone.0014722

[2] Neumann, G., Macken, C.A. and Kawaoka, Y. (2014) Identification of Amino Acid Changes That May Have Been Critical for the Genesis of A(H7N9) Influenza Viruses. Journal of Virology, 88, 4877-4896. http://dx.doi.org/10.1128/JVI.00107-14

[3] Liu, D., Shi, W., Shi, Y., Wang, D., Xiao, H., Li, W., Bi, Y., Wu, Y., Li, X., Yan, J., Liu, W., Zhao, G., Yang, W., Wang, Y., Ma, J., Shu, Y., Lei, F. and Gao, G.F. (2013) Origin and Diversity of Novel Avian Influenza A H7N9 Viruses Causing Human Infection: Phylogenetic, Structural, and Coalescent Analyses. Lancet, 381, 1926-1932. http://dx.doi.org/10.1016/S0140-6736(13)60938-1

[4] Du Ry van Beest Holle, M., Meijer, A., Koopmans, M. and de Jager, C.M. (2005) Human-to-Human Transmission of Avian Influenza A/H7N7, the Netherlands, 2003. Euro Surveillance, 10, 584. http://www.eurosurveillance.org/ViewArticle.aspx?ArticleId=584

[5] Banks, J., Speidel, E.S., Moore, E., Plowright, L., Piccirillo, A., et al. (2001) Changes in the Haemagglutinin and the Neuraminidase Genes Prior to the Emergence of Highly Pathogenic H7N1 Avian Influenza Viruses in Italy. Archives of Virology, 146, 963-973. http://dx.doi.org/10.1007/s007050170128

[6] Campitelli, L., Mogavero, E., De Marco, M.A., Delogu, M., Puzelli, S., et al. (2004) Interspecies Transmission of an H7N3 Influenza Virus from Wild Birds to Intensively Reared Domestic Poultry in Italy. Virology, 323, 24-36. http://dx.doi.org/10.1016/j.virol.2004.02.015

[7] Blumenkrantz, D., Roberts, K.L., Shelton, H., Lycett, S. and Barclay, W.S. (2013) The Short Stalk Length of Highly Pathogenic Avian Influenza H5N1 Virus Neuraminidase Limits Transmission of Pandemic H1N1 Virus in Ferrets. Journal of Virology, 87, 10539-10551. http://dx.doi.org/10.1128/JVI.00967-13

[8] Cosic, I. (1997) The Resonant Recognition Model of Macromolecular Bioreactivity, Theory and Application. Birkhauser Verlag, Berlin. http://dx.doi.org/10.1007/978-3-0348-7475-5

[9] Veljkovic, V., Niman, H.L., Glisic, S., et al. (2009) Identification of Hemag-Glutinin Structural Domain and Polymorphisms Which May Modulate Swine H1N1 Interactions with Human Receptor. BMC Structural Biology, 9, 62. http://dx.doi.org/10.1186/1472-6807-9-62

[10] Veljkovic, V., Veljkovic, N., Muller, C.P., et al. (2009) Characterization of Conserved Properties of Hemagglutinin of H5N1 and Human Influenza Viruses: Possible Consequences for Therapy and Infection Control. BMC Structural Biology, 7, 9-21.

[11] Hu, W. (2013) Receptor Binding Specificity and Sequence Comparison of a Novel Avian-Origin H7N9 Virus in China. Journal of Biomedical Science and Engineering, 6, 533-542. http://dx.doi.org/10.4236/jbise.2013.65068

[12] Hu, W. (2013) Mutations in Hemagglutinin of a Novel Avian-Origin H7N9 Virus That Are Critical for Receptor Binding Specificity. Tsinghua Science and Technology, 18, 522-529. http://dx.doi.org/10.1109/TST.2013.6616525

[13] Hu, W. (2014) Functional Interplay between Hemagglutinin and Neuraminidase of Pandemic 2009 H1N1 from the Perspective of Virus Evolution. In: Basu, M., Pan, Y. and Wang, J., Eds., Lecture Notes in Computer Science, Vol. 8492, Springer, Berlin, 38-49.

[14] Shi, Y., Zhang, W., Wang, F., Qi, J., Wu, Y., Song, H., Gao, F., Bi, Y., Zhang, Y., Fan, Z., Qin, C., Sun, H., Liu, J., Haywood, J., Liu, W., Gong, W., Wang, D., Shu, Y., Wang, Y., Yan, J. and Gao, G.F. (2013) Structures and Receptor 
Binding of Hemagglutinins from Human-Infecting H7N9 Influenza Viruses. Science, 342, 243-247. http://dx.doi.org/10.1126/science.1242917

[15] Xiong, X., Martin, S.R., Haire, L.F., Wharton, S.A., Daniels, R.S., Bennett, M.S., McCauley, J.W., Collins, P.J., Walker, P.A., Skehel, J.J. and Gamblin, S.J. (2013) Receptor Binding by an H7N9 Influenza Virus from Humans. Nature, 499, 496-499. http://dx.doi.org/10.1038/nature12372

[16] Xu, R., de Vries, R.P., Zhu, X., Nycholat, C.M., McBride, R., Yu, W., Paulson, J.C. and Wilson, I.A. (2013) Preferential Recognition of Avian-Like Receptors in Human Influenza A H7N9 Viruses. Science, 342, 1230-1235. http://dx.doi.org/10.1126/science.1243761

[17] Zhu, H., Wang, D., Kelvin, D.J., Li, L., Zheng, Z., Yoon, S.W., Wong, S.S., Farooqui, A., Wang, J., Banner, D., Chen, R., Zheng, R., Zhou, J., Zhang, Y., Hong, W., Dong, W., Cai, Q., Roehrl, M.H., Huang, S.S., Kelvin, A.A., Yao, T., Zhou, B., Chen, X., Leung, G.M., Poon, L.L., Webster, R.G., Webby, R.J., Peiris, J.S., Guan, Y. and Shu, Y. (2013) Infectivity, Transmission, and Pathology of Human-Isolated H7N9 Influenza in Ferrets and Pigs. Science, 341, 183186. http://dx.doi.org/10.1126/science.1239844

[18] Watanabe, T., Kiso, M., Fukuyama, S., Nakajima, N., Imai, M., Yamada, S., Murakami, S., et al. (2013) Characterization of H7N9 Influenza A Viruses Isolated from Humans. Nature, 501, 551-555. http://dx.doi.org/10.1038/nature12392

[19] Belser, J.A., Gustin, K.M., Pearce, M.B., Maines, T.R., Zeng, H., Pappas, C., Sun, X., Carney, P.J., Villanueva, J.M., Stevens, J., Katz, J.M. and Tumpey, T.M. (2013) Pathogenesis and Transmission of Avian Influenza A (H7N9) Virus in Ferrets and Mice. Nature, 501, 556-559. http://dx.doi.org/10.1038/nature12391

[20] Ramos, I., Krammer, F., Hai, R., Aguilera, D., Bernal-Rubio, D., Steel, J., García-Sastre, A. and Fernandez-Sesma, A (2013) H7N9 Influenza Viruses Interact Preferentially with $\alpha 2,3$-Linked Sialic Acids and Bind Weakly to $\alpha 2,6$-Linked Sialic Acids. Journal of General Virology, 94, 2417-2423. http://dx.doi.org/10.1099/vir.0.056184-0

[21] Jonges, M., Bataille, A., Enserink, R., Meijer, A., Fouchier, R.A., Stegeman, A., Koch, G. and Koopmans, M. (2011) Comparative Analysis of Avian Influenza Virus Diversity in Poultry and Humans during a Highly Pathogenic Avian Influenza A (H7N7) Virus Outbreak. Journal of Virology, 85, 10598-10604. http://dx.doi.org/10.1128/JVI.05369-11

[22] Neumann, G., Macken, C.A. and Kawaoka, Y. (2014) Identification of Amino Acid Changes that May Have Been Critical for the Genesis of A(H7N9) Influenza Viruses. Journal of Virology, 88, 4877-4896. 
Scientific Research Publishing (SCIRP) is one of the largest Open Access journal publishers. It is currently publishing more than 200 open access, online, peer-reviewed journals covering a wide range of academic disciplines. SCIRP serves the worldwide academic communities and contributes to the progress and application of science with its publication.

Other selected journals from SCIRP are listed as below. Submit your manuscript to us via either submit@scirp.org or Online Submission Portal.
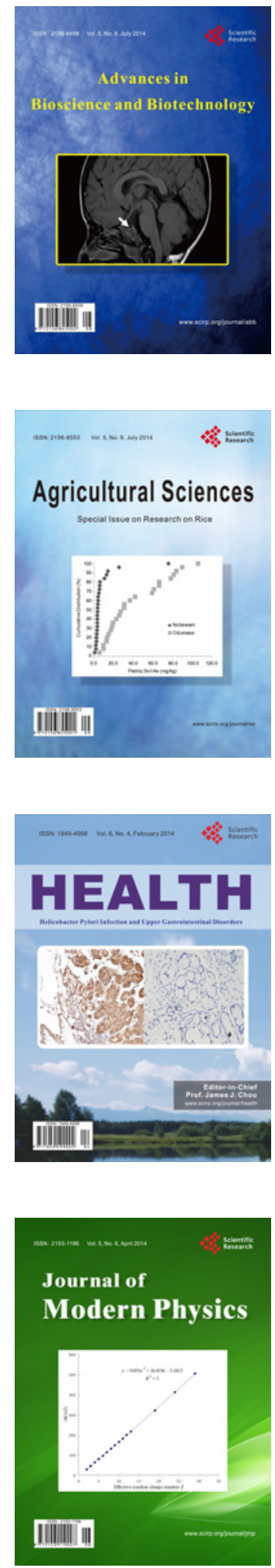
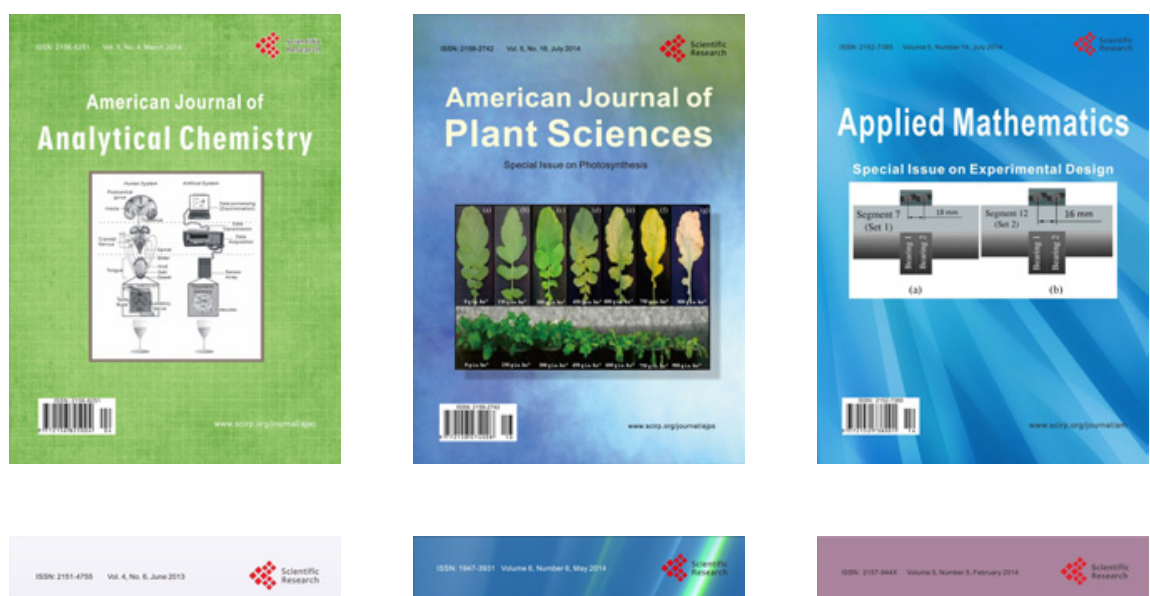

Creative Education
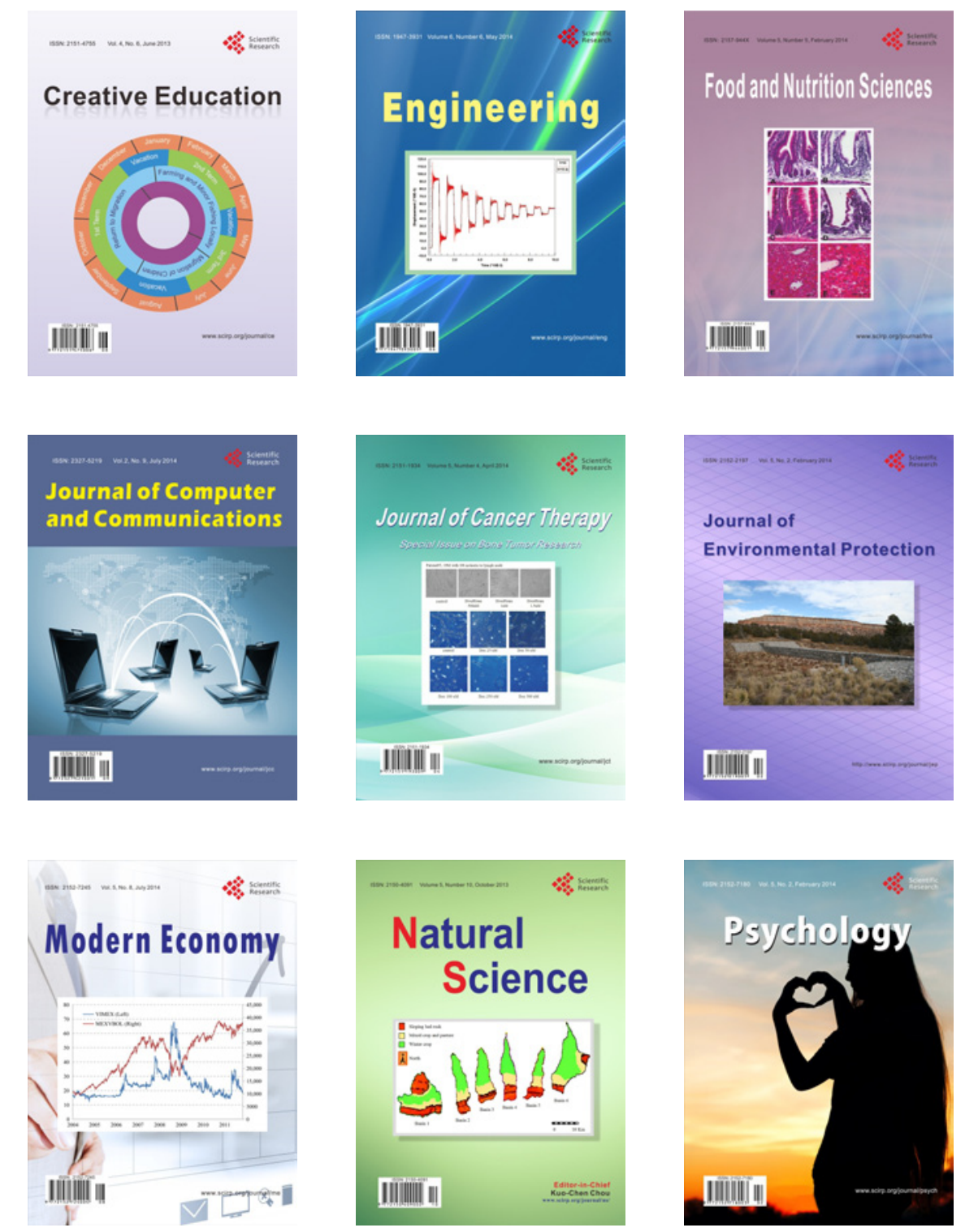